\title{
The survival and clinicopathological differences between patients with stage IIIA and stage II rectal cancer: An analysis of 12,036 patients in the SEER database
}

\author{
Ben Huang ${ }^{1, *}$, Shaobo Mo ${ }^{1,},{ }^{*}$, Liang Zhu ${ }^{1}$, Tianhong $\mathbf{X u}^{1}$, Guoxiang Cai ${ }^{1}$ \\ ${ }^{1}$ Department of Colorectal Surgery, Fudan University Shanghai Cancer Center, Shanghai 20032, People's Republic of China \\ *These authors have contributed equally to this work \\ Correspondence to: Guoxiang Cai, email: gxcfuscc@163.com \\ Keywords: rectal cancer, stage IIIA, stage II, survival \\ Received: August 02, $2016 \quad$ Accepted: October 17, $2016 \quad$ Published: October 28, 2016
}

\section{ABSTRACT}

Background: Stage IIIA rectal cancer has distinctive oncological features, including limited depth of intestinal wall invasion and early regional lymph node metastasis. We aim to compare survival outcomes and clinicopathological features for stage IIIA rectal cancer with those for stage II rectal cancer.

Method: We analyzed patients with stage II or stage IIIA rectal cancer treated with surgery without receiving preoperative radiotherapy based on data from the US Surveillance, Epidemiology, and End Results (SEER) database between 1988 and 2003. Survival curves were plotted using the Kaplan-Meier method. Multivariate Cox proportional analyses were utilized to analyze independent prognostic factors for cancer-specific survival (CSS).

Results: We included 12,036 rectal cancer patients $(10,132$ stage II and 1,904 stage IIIA) from the SEER database. Patients with stage IIIA rectal cancer had smaller tumor size than patients with stage II rectal cancer. A multivariate analysis suggested that compared with patients with stage IIIA rectal cancer, patients with stage II disease were more likely to have more unfavorable CSS (HR 1.195, 95\% CI 1.079-1.324, $\mathrm{p}=0.001$ ). When stage II rectal cancer was further analyzed as stage IIA, IIB and IIC rectal cancer, the multivariate analysis indicated that compared with patients with stage IIIA rectal cancer, patients with stage IIA rectal cancer (HR $1.113,95 \%$ CI 1.003-1.235, $p=0.044)$, stage IIB rectal cancer (HR $1.493,95 \%$ CI 1.267-1.758, p<0.001) and stage IIC rectal cancer (HR 2.712, 95\% CI 2.319-3.171, $\mathrm{p}<0.001$ ) were also more likely to exhibit more unfavorable CSS.

Conclusion: Patients with stage IIIA rectal cancer had more favorable survival outcomes and smaller tumor size compared with patients with stage II rectal cancer.

\section{INTRODUCTION}

Rectal cancer is one of the most commonly diagnosed malignancies worldwide, with approximately 40 thousand new cases predicted to occur in the United States in 2016 [1]. The tumor, node, and metastasis (TNM) classification is currently the most frequently used rectal cancer staging system; it has been progressively updated to better tailor therapeutic strategies and predict oncologic outcomes [2-5]. In the 7th edition of the AJCC cancer staging system, tumors with lymph node metastasis, except for those related to metastatic rectal cancer, were divided into stage IIIA, IIIB and IIIC subgroups according to heterogeneous survival outcomes [6].

Stage IIIA rectal cancer is defined as tumors that invade submucosa with metastasis in 1-6 regional lymph nodes (T1N1-N2a) and muscularis propria with involvement of 1-3 regional lymph nodes (T2N1) [7]. Patients with stage IIIA rectal cancer were reported to have better survival outcomes compared with patients with other subgroups of stage III disease; the 5-year observed survival rates for stage III subcategories were $55 \%$ for stage IIIA, $35 \%$ for stage IIIB and $24 \%$ for stage IIIC [8]. Gunderson et al.[9] analyzed 35,829 patients 
from the SEER database and found that patients with T1-2N1/T1N2a rectal cancer had a 5-year observed survival rate comparable with patients with T2N0 disease (72\%/73\% vs. $75 \%$, respectively). Li et al.[10] suggested that the 5-year overall survival of patients with stage IIIA colorectal cancer $(86 \%)$ was greater than that of patients with stage II disease (75\%), and comparable with that of patients with stage I disease (90\%).

Multimodality therapy, i.e., preoperative chemoradiotherapy (CRT) followed by curative surgery, has been established as the standard treatment for patients with locally advanced rectal cancer during the last decade $[11,12]$. Preoperative CRT followed by total mesorectal excision (TME) was reported to result in a decline in local recurrence and an improvement in oncologic outcomes in patients with cT3/T4 or node-positive rectal cancer [13]. Most patients respond to preoperative CRT, which downstages the tumor and leads to a considerable reduction in tumor burden, and almost $10 \%-30 \%$ of patients obtain a pathologic complete response (pCR) in their rectal cancer [14-16]. For patients with locally advanced rectal cancer with medical contraindication to multimodality therapy, CRT is also recommended as the standard adjuvant therapeutic strategy by National Institute of Health [17, 18].

Patients with stage IIIA colorectal cancer are reported to have a favorable prognosis; however, comparisons of survival outcomes and clinicopathological features between stage II and stage IIIA rectal cancer have rarely been performed. Here, we conduct an analysis that compares the survival and clinicopathological differences between patients with stage II and stage IIIA rectal cancer based on patient records in the US Surveillance, Epidemiology, and End Results (SEER) database.

\section{RESULTS}

\section{Descriptive statistics}

We included 12,036 rectal cancer patients $(10,132$ stage II and 1,904 stage IIIA) in the analysis. In total, 3,891 $(32.3 \%)$ rectal cancer-specific deaths were identified. The median follow-up duration was 88 months (interquartile range, 36-126 months). Clinicopathological characteristics of the stage II and stage IIIA rectal cancer patients are shown in Table 1. The entire sample was comprised of $6,675(55.5 \%)$ men and 5,361 (44.5\%) women. The sample was predominantly Caucasian $(84.3 \%)$, followed by African-American (7.0\%). Histological types included adenocarcinoma $(91.9 \%)$, mucinous cancer $(7.8 \%)$, and signet-ring cell cancer $(0.3 \%)$. Patients with stage II rectal cancer tended to be older than patients with stage IIIA disease $(p<0.001)$. Cases with stage II rectal cancer had larger mean tumor size compared with cases with stage IIIA lesions.

\section{Stage II vs. stage IIIA}

Kaplan-Meier analysis suggested that patients with stage IIIA rectal cancer had a more favorable CSS compared with patients with stage II disease $(p<0.001$, Figure 1); the 5-year CSS of patients with stage IIIA and stage II rectal cancer was 80\% (95\% CI 80\%-80\%) and $73 \%$ (95\% CI 72\%-73\%), respectively. KaplanMeier analyses of the entire cohort indicated that age at diagnosis $(\mathrm{p}<0.001)$, race $(\mathrm{p}<0.001)$, histological type $(p<0.001)$, grade of differentiation $(p<0.001)$, number of lymph nodes harvested $(\mathrm{LNH})(\mathrm{p}<0.001)$ and TNM stage $(\mathrm{p}<0.001)$ were risk factors associated with CSS (Table 2). Univariate Cox proportional hazard regression demonstrated factors associated with CSS were age at diagnosis $(p<0.001)$, race $(p<0.001)$, histological type $(p<0.001)$, grade of differentiation $(p<0.001)$, LNH $(\mathrm{p}<0.001)$ and TNM stage $(\mathrm{p}<0.001)$. An analysis using the multivariate Cox proportional model identified the following independent prognostic factors for CSS: age at diagnosis $(\mathrm{p}<0.001)$, race $(\mathrm{p}<0.001)$, histological type $(\mathrm{p}=0.009)$, grade of differentiation $(\mathrm{p}<0.001)$, tumor size $(p<0.001)$, LNH $(p<0.001)$, and TNM stage $(p=0.001)$ (Table 3 ). Compared with patients with stage IIIA rectal cancer, patients with stage II disease were more likely to have more unfavorable CSS (HR 1.195, 95\% CI 1.079$1.324, \mathrm{p}=0.001)$. Kaplan-Meier curve of overall survival for patients with stage II and stage IIIA rectal cancer was shown in Figure $\mathrm{S} 1(\mathrm{p}<0.001)$.

Patients with stage II rectal cancer was further analyzed as patients with stage IIA, IIB and IIC rectal cancer. Kaplan-Meier analysis showed a significant difference in CSS in patients with stage IIA, IIB, stage IIC and stage IIIA rectal cancer $(\mathrm{p}<0.001$, Figure 2$)$; the 5-year CSS for patients with stage IIA, stage IIB, stage IIC and stage IIIA rectal cancer was 75\% $(95 \%$ CI 75\%-75\%), 67\% (95\% CI 66\%-67\%), 46\% (95\% CI 45\%-46\%) and 80\% (95\% CI 80\%-80\%), respectively. Multivariate analysis identified TNM stage $(\mathrm{p}<0.001)$ as an independent prognostic factor for CSS (Table 4). Compared with patients with stage IIIA rectal cancer, patients with stage IIA rectal cancer (HR 1.113, 95\% CI 1.003-1.235, $\mathrm{p}=0.044)$, stage IIB rectal cancer (HR 1.493, 95\% CI 1.267-1.758, $\mathrm{p}<0.001)$ and stage IIC rectal cancer (HR 2.712, 95\% CI 2.319-3.171, $\mathrm{p}<0.001$ ) were more likely to have more unfavorable CSS. Kaplan-Meier curve of overall survival for patients with stage IIA, stage IIB, stage IIC and stage IIIA rectal cancer was shown in Figure $\mathrm{S} 2(\mathrm{p}<0.001)$.

\section{DISCUSSION}

The criterion that differentiates stage III disease from stage II disease is the presence or absence of lymph node metastasis based on the AJCC cancer staging system 
Table 1: Demographics of patients with stage II and stage IIIA rectal cancer from the SEER database [N (\%)]

\begin{tabular}{|c|c|c|c|c|}
\hline \multirow{2}{*}{ Characteristics } & Total & II & IIIA & \multirow{2}{*}{ P value } \\
\hline & $(\mathrm{N}=12036)$ & $(\mathrm{N}=10132)$ & $(\mathrm{N}=1904)$ & \\
\hline Median follow-up (mos) & 88 & 85 & 98 & \\
\hline Mean tumor size $(\mathrm{cm})$ & 4.8 & 5.0 & 3.5 & \\
\hline Sex & & & & 0.313 \\
\hline Male & $6675(55.5)$ & $5599(55.3)$ & $1076(56.5)$ & \\
\hline Female & $5361(44.5)$ & $4533(44.7)$ & $828(43.5)$ & \\
\hline Median age at diagnosis & 69 & 69 & 65 & \\
\hline IQR & $59-77$ & $59-78$ & $55-74$ & \\
\hline Age at diagnosis (yrs) & & & & $<0.001$ \\
\hline$\leq 70$ & $6674(55.5)$ & $5421(53.5)$ & $1253(65.8)$ & \\
\hline$>70$ & $5362(44.5)$ & $4711(46.5)$ & $651(34.2)$ & \\
\hline Race & & & & 0.030 \\
\hline White & $10147(84.3)$ & $8566(84.6)$ & $1581(83.0)$ & \\
\hline Black & $838(7.0)$ & $711(7.0)$ & $127(6.7)$ & \\
\hline Other $^{\text {a }}$ & $1051(8.7)$ & $855(8.4)$ & $196(10.3)$ & \\
\hline Histological Type & & & & 0.296 \\
\hline Adenocarcinoma & $11055(91.9)$ & $9291(91.7)$ & $1764(92.6)$ & \\
\hline Mucinous adenocarcinoma & $943(7.8)$ & $810(8.0)$ & $133(7.0)$ & \\
\hline Signet-ring cell carcinoma & $38(0.3)$ & $31(0.3)$ & $7(0.4)$ & \\
\hline Grade of differentiation & & & & $<0.001$ \\
\hline Well/Moderate & $10425(86.6)$ & $8851(87.4)$ & $1574(82.7)$ & \\
\hline Poor/Undifferentiated & $1611(13.4)$ & $1281(12.6)$ & $330(17.3)$ & \\
\hline Median LNH & 8 & 8 & 8 & \\
\hline IQR & $5-14$ & $4-14$ & $5-13$ & \\
\hline LNH & & & & 0.009 \\
\hline$<12$ & $7938(66.0)$ & $6633(65.5)$ & $1305(68.5)$ & \\
\hline$\geq 12$ & $4098(34.0)$ & $3499(34.5)$ & $599(31.5)$ & \\
\hline
\end{tabular}

LNH $=$ number of lymph nodes harvested.

${ }^{a}$ Includes Native American, Asian, Pacific Islander and Unknown.

for rectal cancer. Because of the contradictory oncologic features of stage IIIA rectal cancer, including shallow intestinal wall invasion (T1/T2) and early regional lymph node metastasis, patients with stage IIIA rectal cancer may theoretically exhibit conflicting survival outcomes. On one hand, the disease possesses a relatively limited depth of intestinal wall invasion and metastasis is confined to regional lymph nodes around the primary tumor. Disease in this stage should be easily resected with curative intent and is generally regarded as exhibiting a favorable 
prognosis. On the other hand, early metastasis to regional lymph nodes with a limited intestinal wall invasion may suggest the aggressive nature of these lesions, which may result in a decreased prognosis [7]. Actually, increased oncologic outcomes have been observed in stage IIIA colorectal cancer compared with the other subgroups of stage III disease, and Mukai et al.[19] suggested that the T1/2N1 category of colorectal cancer should be redefined as stage I or stage II colorectal cancer.

The results from our study suggested that patients with stage IIIA rectal cancer had more favorable CSS than patients with stage II rectal cancer. However, the hazard ratio (1.113) between stage IIA and stage IIIA rectal cancer is quite small, therefore, the clinical significance of survival paradox between stage IIA and stage IIIA rectal cancer may be limited. In surgical procedures of colorectal cancer, every effort should be done to achieve a negative margin. Chu et al.[20] indicated that for patients with stage IIIA colon cancer, only $1 \%$ had residual tumor compared with $19 \%$ for patients with stage IIB/C colon cancer $(p<0.0001)$, the positive surgical margins may contribute to the survival contradiction between patients with stage $\mathrm{IIB} / \mathrm{C}$ and stage IIIA colon cancer. The results from our study revealed that patients with stage IIIA rectal cancer had smaller tumor size than patients with stage II disease. For a T1/T2 tumor with small tumor size (i.e., stage IIIA), en bloc resection of the primary lesion should be easier to be accomplished than for a T3/T4 tumor with larger tumor size (i.e., stage II) to achieve negative margins status. In addition, surgical proficiency of the operation on locally advanced rectal cancer may vary among different surgeons, leading to a greater rate of positive margin in stage II rectal cancer than in stage IIIA disease [20]. The positive surgical margins may explain the survival paradox between stage IIIA and stage II rectal cancer.

The obvious defect of the current AJCC cancer staging system in colorectal cancer is the relatively overestimated weighting of node metastasis ( $\mathrm{N}$ stage) $[10,21]$. In malignancies such as esophageal, gastric, breast and lung cancer, stage II tumors are defined by both $\mathrm{T}$ category (T1/2) and N category (N1), according to the AJCC cancer staging system. However, in colorectal cancer, apart from patients with distal metastasis (stage IV), all node-positive patients are categorized as stage III regardless of their $\mathrm{T}$ status based on the TNM classification [5]. Although lymph node status has been identified as an essential prognostic factor in colorectal cancer that can guide adjuvant therapy and evaluate prognosis [22, 23], the integration of TN categories into a cancer staging system remains complicated. Therefore, the over-estimated weighting of node metastasis in the current AJCC cancer staging system leads to poor monotonicity of gradients from the early to the advanced cancer stages in colorectal cancer [24, 25]. Some researchers have proposed an improved AJCC cancer staging system, in which stage IIIA disease is reclassified as stage I or stage II based on

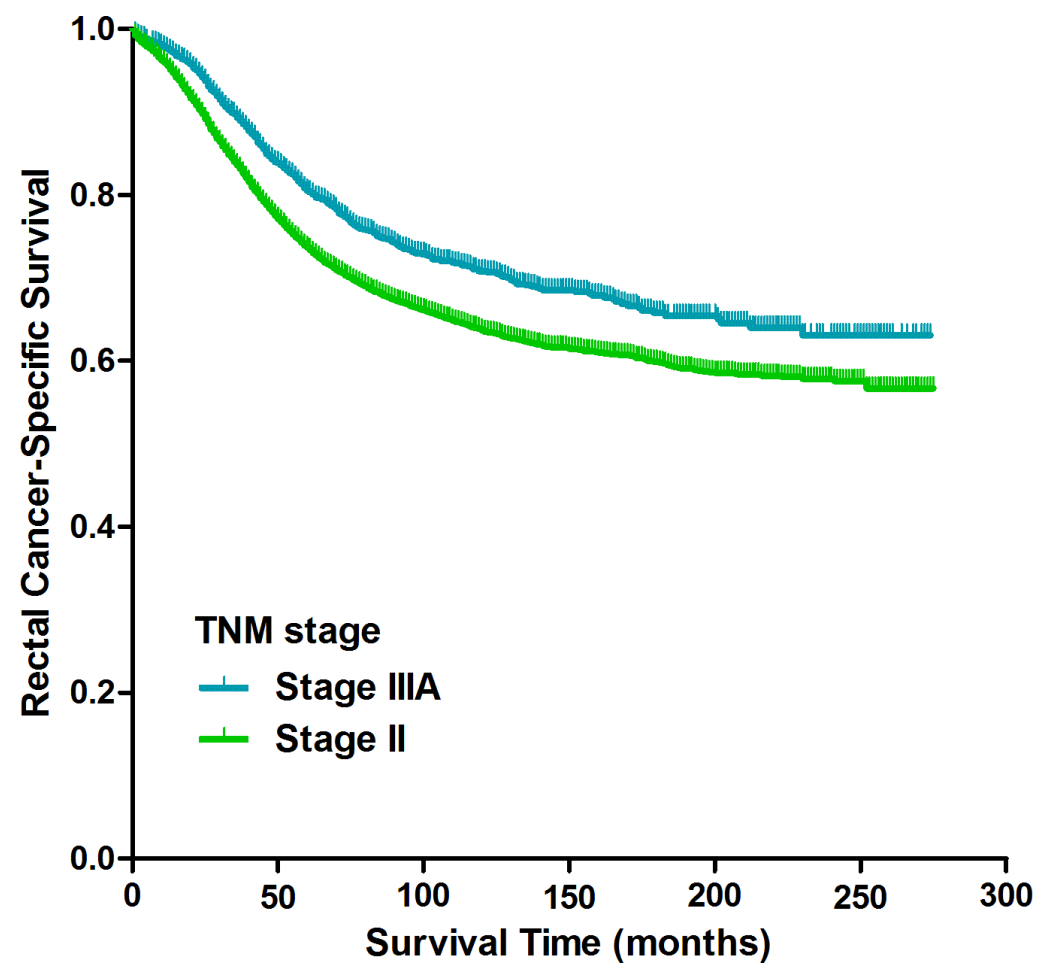

Figure 1: Kaplan-Meier curves of cancer-specific survival for patients with stage II and stage IIIA rectal cancer from the SEER database. 
Table 2: Univariate survival analyses of patients with stage II and stage IIIA rectal cancer from the SEER database

\begin{tabular}{|c|c|c|c|c|}
\hline Variable & No. & 5-year CSS & $\log \operatorname{rank} \chi^{2}$ & P-value \\
\hline Sex & & & 1.545 & 0.214 \\
\hline Male & 6675 & $74 \%$ & & \\
\hline Female & 5361 & $74 \%$ & & \\
\hline Age at diagnosis (yrs) & & & 294.925 & $<0.001$ \\
\hline$\leq 70$ & 3494 & $79 \%$ & & \\
\hline$>70$ & 8542 & $66 \%$ & & \\
\hline Race & & & 57.126 & $<0.001$ \\
\hline White & 10147 & $74 \%$ & & \\
\hline Black & 838 & $65 \%$ & & \\
\hline Other ${ }^{a}$ & 1051 & $80 \%$ & & \\
\hline Histological Type & & & 32.868 & $<0.001$ \\
\hline Adenocarcinoma & 11055 & $75 \%$ & & \\
\hline Mucinous adenocarcinoma & 943 & $66 \%$ & & \\
\hline Signet-ring cell carcinoma & 38 & $54 \%$ & & \\
\hline Grade of differentiation & & & 14.664 & $<0.001$ \\
\hline Well/Moderate & 10425 & $75 \%$ & & \\
\hline Poor/Undifferentiated & 1611 & $69 \%$ & & \\
\hline LNH & & & 95.038 & $<0.001$ \\
\hline$<12$ & 7938 & $71 \%$ & & \\
\hline$\geq 12$ & 4098 & $80 \%$ & & \\
\hline TNM stage & & & 41.709 & $<0.001$ \\
\hline IIIA & 1904 & $80 \%$ & & \\
\hline II & 10132 & $73 \%$ & & \\
\hline TNM stage $^{\mathrm{b}}$ & & & 364.717 & $<0.001$ \\
\hline IIIA & 1904 & $80 \%$ & & \\
\hline IIA & 8852 & $75 \%$ & & \\
\hline IIB & 693 & $67 \%$ & & \\
\hline IIC & 587 & $46 \%$ & & \\
\hline
\end{tabular}

CSS $=$ cancer-specific survival, $\mathrm{LNH}=$ number of lymph nodes harvested.

${ }^{a}$ Includes Native American, Asian, Pacific Islander and Unknown.

${ }^{\mathrm{b}}$ Stage II rectal cancer was analyzed as Stage IIA, IIB and IIC rectal cancer.

a cluster analysis of TN scores in colorectal cancer [24]. The favorable oncological outcomes of stage IIIA rectal cancer indicate that weighting of the T status in the TNM staging system may be under-estimated, and conventional notions should be reconsidered according to contemporary survival data in rectal cancer.
According to the National Comprehensive Cancer Network (NCCN) guidelines for rectal cancer, preoperative CRT has been recommended as a crucial therapeutic strategy for locally advanced rectal cancer. Growing evidence suggests that patients with locally advanced rectal cancer benefit from preoperative CRT 
Table 3: Multivariate survival analyses of patients with stage II and stage IIIA rectal cancer from the SEER database

\begin{tabular}{|c|c|c|c|}
\hline \multirow{2}{*}{ Variable } & \multicolumn{2}{|c|}{ Multivariate analysis } & \multirow{2}{*}{ P value } \\
\hline & HR & $95 \%$ CI & \\
\hline Age at diagnosis (yrs) & & & $<0.001$ \\
\hline$\leq 70$ & 1 & reference & \\
\hline$>70$ & 1.686 & $1.572-1.807$ & \\
\hline Race & & & $<0.001$ \\
\hline White & 1 & reference & \\
\hline Black & 1.450 & $1.283-1.639$ & $<0.001$ \\
\hline Other ${ }^{\text {a }}$ & 0.767 & $0.671-0.877$ & $<0.001$ \\
\hline Histological Type & & & 0.009 \\
\hline Adenocarcinoma & 1 & reference & \\
\hline Mucinous adenocarcinoma & 1.195 & $1.054-1.355$ & 0.006 \\
\hline Signet-ring cell carcinoma & 1.492 & $0.823-2.704$ & 0.187 \\
\hline Grade of differentiation & & & $<0.001$ \\
\hline Well/Moderate & 1 & reference & \\
\hline Poor/Undifferentiated & 1.207 & $1.095-1.329$ & \\
\hline Tumor size $(\mathrm{cm})$ & 1.022 & $1.014-1.031$ & $<0.001$ \\
\hline LNH & & & $<0.001$ \\
\hline$<12$ & 1 & reference & \\
\hline$\geq 12$ & 0.723 & $0.670-0.779$ & \\
\hline TNM stage & & & 0.001 \\
\hline IIIA & 1 & reference & \\
\hline II & 1.195 & $1.079-1.324$ & \\
\hline
\end{tabular}

$\mathrm{HR}=$ hazard ratio, $\mathrm{CI}=$ confidence interval, $\mathrm{LNH}=$ number of lymph nodes harvested.

a Includes Native American, Asian, Pacific Islander and Unknown.

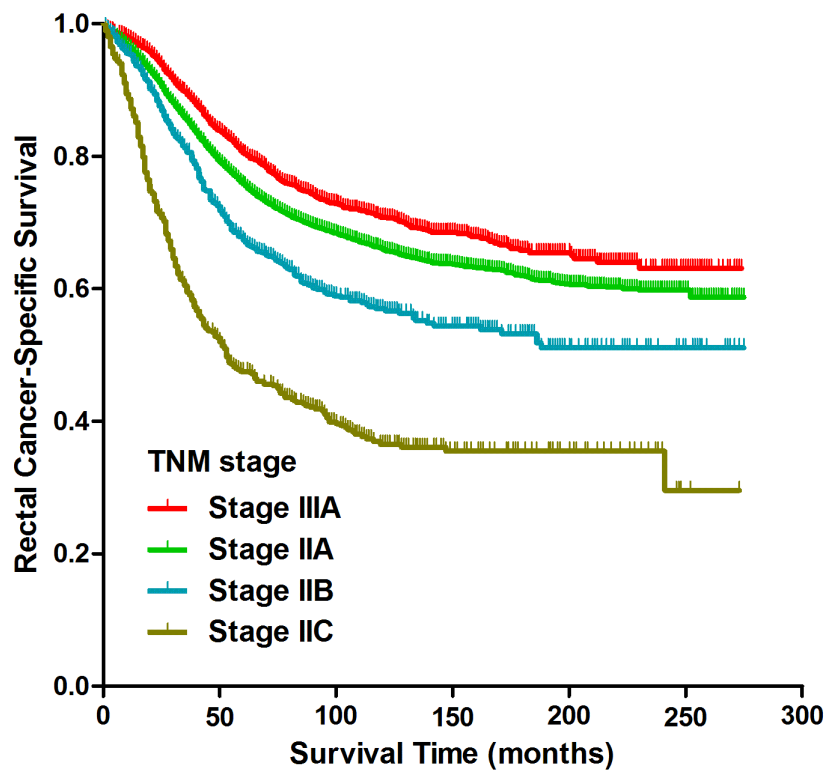

Figure 2: Kaplan-Meier curves of cancer-specific survival for patients with stage IIA, stage IIB, stage IIC and stage IIIA rectal cancer from the SEER database. 
Table 4: Multivariate survival analyses of patients with stage IIA, IIB, IIC and IIIA rectal cancer from the SEER database

\begin{tabular}{|c|c|c|c|}
\hline \multirow{2}{*}{ Variable } & \multicolumn{2}{|c|}{ Multivariate analysis } & \multirow{2}{*}{ P value } \\
\hline & HR & $95 \% \mathrm{CI}$ & \\
\hline Age at diagnosis (yrs) & & & $<0.001$ \\
\hline$\leq 70$ & 1 & reference & \\
\hline$>70$ & 1.691 & $1.577-1.814$ & \\
\hline Race & & & $<0.001$ \\
\hline White & 1 & reference & \\
\hline Black & 1.402 & $1.240-1.585$ & $<0.001$ \\
\hline Other ${ }^{a}$ & 0.753 & $0.659-0.861$ & $<0.001$ \\
\hline Histological Type & & & 0.092 \\
\hline Adenocarcinoma & 1 & reference & \\
\hline Mucinous adenocarcinoma & 1.144 & $1.009-1.298$ & 0.036 \\
\hline Signet-ring cell carcinoma & 1.228 & $0.677-2.227$ & 0.500 \\
\hline Grade of differentiation & & & $<0.001$ \\
\hline Well/Moderate & 1 & reference & \\
\hline Poor/Undifferentiated & 1.201 & $1.090-1.324$ & \\
\hline Tumor size $(\mathrm{cm})$ & 1.018 & $1.009-1.028$ & $<0.001$ \\
\hline LNH & & & $<0.001$ \\
\hline$<12$ & 1 & reference & \\
\hline$\geq 12$ & 0.720 & $0.668-0.777$ & \\
\hline TNM stage & & & $<0.001$ \\
\hline IIIA & 1 & reference & \\
\hline IIA & 1.113 & $1.003-1.235$ & 0.044 \\
\hline IIB & 1.493 & $1.267-1.758$ & $<0.001$ \\
\hline IIC & 2.712 & $2.319-3.171$ & $<0.001$ \\
\hline
\end{tabular}

$\mathrm{HR}=$ hazard ratio, $\mathrm{CI}=$ confidence interval, $\mathrm{LNH}=$ number of lymph nodes harvested.

${ }^{a}$ Includes Native American, Asian, Pacific Islander and Unknown.

in terms of pCR, local disease control and sphincter preservation in comparison with surgery alone or postoperative CRT [13, 26-28]. However, it's still controversial whether preoperative CRT results in sufficient benefits for low-risk patients with stage IIA rectal cancer to outweigh the toxicity, complication, and inconvenience of treatment [29]. Despite this controversy, findings from the current study have led us to recommend preoperative CRT for patients with stage IIA rectal cancer. For patients with locally advanced rectal cancer with medical contraindication to multimodality therapy, adjuvant therapy following surgery is necessary. The Gastrointestinal Tumor Study Group and National Surgical
Adjuvant Breast and Bowel Project Trials demonstrated that for patients with Duke B and Duke C rectal cancer, adjuvant CRT could reduce local recurrence rate and prolong disease-free survival $[30,31]$. Therefore, the 1990 National Institute of Health consensus statement recommended chemotherapy combined with radiotherapy as the standard adjuvant therapeutic strategy for $\mathrm{pT} 3$ or pN1-2 rectal cancer [17]. However, Gunderson et al.[32] showed that for patients with intermediate-risk stage of rectal cancer (T1-2N1, T3N0), adjuvant CRT could not prolong disease-free survival and overall survival compared with adjuvant chemotherapy alone. The use of CRT following surgery in all intermediate-risk stage 
of rectal cancer may be excessive. Risk factors such as tumor location, grade of differentiation, circumferential resection margin, lymphovascular invasion and perineural invasion need to be evaluated for individualized treatment, adjuvant chemotherapy alone may be considered for lowrisk patients with $\mathrm{T} 1-2 \mathrm{~N} 1 / \mathrm{T} 3 \mathrm{~N} 0$ rectal cancer.

To the best of our knowledge, this is the first comprehensive study to reveal favorable prognosis in and distinctive clinicopathological features of patients with stage IIIA rectal cancer. We used the SEER database to ensure a large sample size, and to be specific, our study included of 12,036 total patients, which guaranteed our findings would have adequate power. However, there are still several limitations to our study. One remarkable limitation of the SEER database is that it does not include record of some important patient- and disease-related variables, including lymphovascular or perineural invasion, comorbidities, intestinal obstruction or penetration, circumferential resection margin, and data on adjuvant chemotherapy. These clinicopathological data may be valuable additions to the current study. Another limitation of our study is that only $34.0 \%$ rectal cancer patients had adequate lymph node harvest $(\geq 12)$, for the reason that patients extracted from the SEER database were diagnosed between 1988 and 2003. The definition of tumor deposits (TDs) has changed in the 5th, 6th, and 7 th edition of TNM staging system [3-5]. Nagtegaal et al.[33] revealed that every change in edition of TNM staging system led to a stage migration of between 33\% and $64 \%$ in patients with TDs. Therefore, the changes in the definition of TDs may be a potential limitation in the accuracy of the SEER data. Because information on the clinical staging of rectal cancer patients treated with preoperative radiotherapy is not available, we are unable to compare the survival difference between patients with clinical stage II rectal cancer and clinical stage IIIA rectal cancer treated with preoperative radiotherapy, therefore, our findings may not be generalizable to these patients.

In conclusion, our results provide the first evidence that patients with stage IIIA disease had more favorable survival outcomes and smaller tumor size compared with patients with stage II rectal cancer. Our findings on the favorable prognosis in and distinctive clinicopathological features of patients with stage IIIA rectal cancer are expected to be validated in other institutions.

\section{MATERIALS AND METHODS}

\section{Patient selection}

The SEER database contains 18 cancer registries covering $26 \%$ of the U.S. population and collects and supplies cancer incidence and survival data. We extracted demographic and pathological data for invasive rectal cancer patients between January 1988 and December 2003 from the SEER database (April 2013 release). Patients meeting the following criteria were included in the current analysis: (1) adenocarcinoma, mucinous adenocarcinoma or signet-ring carcinoma of the rectum; (2) known invasion depth and lymph node status; (3) AJCC stage II or stage IIIA; (4) rectal cancer surgically resected with pathology specimen; (5) pathologically confirmed diagnosis; and (6) known survival time and cause of death. Patients were excluded if (1) they received preoperative radiotherapy, (2) they underwent only local tumor excision, (3) their rectal cancer was diagnosed by death certificate or autopsy, or (4) there were other concurrent malignancies. The Fudan University Shanghai Cancer Center Ethical Committee and Institutional Review Board reviewed and approved this study protocol.

\section{Outcome measures}

Records on the following clinicopathological variables were extracted from the SEER database: gender; race; age at and year of diagnosis; primary tumor site; histological type; American Joint Committee on Cancer (AJCC) TNM stage; number of lymph nodes harvested, with 12 as the cutoff value; number of metastatic lymph nodes; depth of intestinal wall invasion; tumor size; grade of differentiation; radiation sequence with surgery; number of primaries; follow-up duration; and SEER classification of cause-specific death. All patients were restaged based on the AJCC cancer stages (7th edition), in which stage IIA was defined as T3N0M0, stage IIB as T4aN0M0, stage IIC as T4bN0M0 and stage IIIA as T1-2N1M0 or T1N2aM0 in rectal cancer. Cancer-specific survival (CSS) from the time of diagnosis to the time of rectal cancerspecific death was the primary outcome. Patients who died from other causes or who were alive at the last follow-up were censored.

\section{Statistical analysis}

Stage II and stage IIIA rectal cancer patient data were summarized using cross-tabulation, and distributions were compared using chi-squared tests. Survival curves were plotted using the Kaplan-Meier method. The log-rank test was used for univariate analysis and variables with a $P$ value less than 0.1 were entered into the multivariate analysis. Multivariate Cox regression analyses were utilized to generate adjusted hazard ratios (HR) and their corresponding 95\% confidence intervals (CIs). Tumor size was analyzed as a continuous variable in the multivariate Cox regression analyses. A two-sided $\mathrm{P}$ value of less than 0.05 was accepted as statistically significant. All statistical analyses were performed using SPSS statistical program version 20.0 (SPSS Inc., Chicago, IL, USA).

\section{ACKNOWLEDGMENTS}

The authors would like to thank SEER program for public access to the database. 


\section{CONFLICTS OF INTEREST}

All authors declare no conflicts of interest.

\section{REFERENCES}

1. Siegel RL, Miller KD, Jemal A. Cancer statistics, 2016. CA Cancer J Clin 2016; 66: 7-30.

2. Quirke P, Williams GT, Ectors N, Ensari A, Piard F, Nagtegaal I. The future of the TNM staging system in colorectal cancer: time for a debate? Lancet Oncol 2007; 8: 651-657.

3. Sobin LH, Fleming ID. TNM Classification of Malignant Tumors, fifth edition (1997). Union Internationale Contre le Cancer and the American Joint Committee on Cancer. Cancer 1997; 80: 1803-1804.

4. Sobin LH. TNM, sixth edition: new developments in general concepts and rules. Semin Surg Oncol 2003; 21: 19-22.

5. Sobin LH, Compton CC. TNM seventh edition: what's new, what's changed: communication from the International Union Against Cancer and the American Joint Committee on Cancer. Cancer 2010; 116: 5336-5339.

6. Edge SB, Compton CC. The American Joint Committee on Cancer: the 7th edition of the AJCC cancer staging manual and the future of TNM. Ann Surg Oncol 2010; 17: 1471-1474.

7. Park SY, Choi GS, Park JS, Kim HJ, Sakai Y, Hasegawa S, Watanabe T, Kim SH. Distinctive oncological features of stage IIIA colorectal cancer: Analysis of prognostic factors for selective adjuvant chemotherapy. J Surg Oncol 2015; 111: 882-890.

8. Greene FL, Stewart AK, Norton HJ. New tumor-nodemetastasis staging strategy for node-positive (stage III) rectal cancer: an analysis. J Clin Oncol 2004; 22: 1778-1784.

9. Gunderson LL, Jessup JM, Sargent DJ, Greene FL, Stewart A. Revised tumor and node categorization for rectal cancer based on surveillance, epidemiology, and end results and rectal pooled analysis outcomes. J Clin Oncol 2010; 28: 256-263.

10. Li J, Yi CH, Hu YT, Li JS, Yuan Y, Zhang SZ, Zheng S, Ding KF. TNM Staging of Colorectal Cancer Should be Reconsidered According to Weighting of the $\mathrm{T}$ Stage: Verification Based on a 25-Year Follow-Up. Medicine (Baltimore) 2016; 95: e2711.

11. Weiser MR, Zhang Z, Schrag D. Locally advanced rectal cancer: time for precision therapeutics. Am Soc Clin Oncol Educ Book 2015; e192-196.

12. Fleming FJ, Pahlman L, Monson JR. Neoadjuvant therapy in rectal cancer. Dis Colon Rectum 2011; 54: 901-912.

13. Kapiteijn E, Marijnen CA, Nagtegaal ID, Putter H, Steup WH, Wiggers T, Rutten HJ, Pahlman L, Glimelius B, van Krieken JH, Leer JW, van de Velde CJ. Preoperative radiotherapy combined with total mesorectal excision for resectable rectal cancer. N Engl J Med 2001; 345: 638-646.

14. Chan AK, Wong AO, Langevin J, Jenken D, Heine J, Buie D, Johnson DR. Preoperative chemotherapy and pelvic radiation for tethered or fixed rectal cancer: a phase II dose escalation study. Int J Radiat Oncol Biol Phys 2000; 48: 843-856.

15. Latkauskas T, Pauzas H, Gineikiene I, Janciauskiene R, Juozaityte E, Saladzinskas Z, Tamelis A, Pavalkis D. Initial results of a randomized controlled trial comparing clinical and pathological downstaging of rectal cancer after preoperative short-course radiotherapy or long-term chemoradiotherapy, both with delayed surgery. Colorectal Dis 2012; 14: 294-298.

16. Bosset JF, Calais G, Mineur L, Maingon P, RadosevicJelic L, Daban A, Bardet E, Beny A, Briffaux A, Collette L. Enhanced tumorocidal effect of chemotherapy with preoperative radiotherapy for rectal cancer: preliminary results--EORTC 22921. J Clin Oncol 2005; 23: 5620-5627.

17. NIH consensus conference. Adjuvant therapy for patients with colon and rectal cancer. Jama 1990; 264: 1444-1450.

18. Trakarnsanga A, Ithimakin S, Weiser MR. Treatment of locally advanced rectal cancer: controversies and questions. World J Gastroenterol 2012; 18: 5521-5532.

19. Mukai M, Kishima K, Fukumitsu H, Sekido Y, Izumi H, Hoshikawa T, Tajima T, Tobita K, Sadahiro S, Yasuda S, Ogoshi $\mathrm{K}$. Is the T1/2N1 ( $</=3$ nodes) category actually stage IIIA (TNM)/IIIa (Japanese classification) in patients with primary colorectal cancer? Oncol Rep 2011; 26: 209-214.

20. Chu QD, Zhou M, Medeiros K, Peddi P. Positive surgical margins contribute to the survival paradox between patients with stage IIB/C (T4N0) and stage IIIA (T1-2N1, T1N2a) colon cancer. Surgery 2016;

21. Mori T. A comparison of the new (planned) TNM classification and Japanese general rule for staging colorectal cancer. Cancer Invest 2010; 28: 387-392.

22. Kotake K, Honjo S, Sugihara K, Hashiguchi Y, Kato T, Kodaira S, Muto T, Koyama Y. Number of lymph nodes retrieved is an important determinant of survival of patients with stage II and stage III colorectal cancer. Jpn J Clin Oncol 2012; 42: 29-35.

23. Chang GJ, Rodriguez-Bigas MA, Skibber JM, Moyer VA. Lymph node evaluation and survival after curative resection of colon cancer: systematic review. J Natl Cancer Inst 2007; 99: 433-441

24. Li J, Guo BC, Sun LR, Wang JW, Fu XH, Zhang SZ, Poston G, Ding KF. TNM staging of colorectal cancer should be reconsidered by $\mathrm{T}$ stage weighting. World J Gastroenterol 2014; 20: 5104-5112.

25. Lan YT, Yang SH, Chang SC, Liang WY, Li AF, Wang HS, Jiang JK, Chen WS, Lin TC, Lin JK. Analysis of the seventh edition of American Joint Committee on colon cancer staging. Int J Colorectal Dis 2012; 27: 657-663. 
26. van Gijn W, Marijnen CA, Nagtegaal ID, Kranenbarg EM, Putter H, Wiggers T, Rutten HJ, Pahlman L, Glimelius B, van de Velde CJ. Preoperative radiotherapy combined with total mesorectal excision for resectable rectal cancer: 12-year follow-up of the multicentre, randomised controlled TME trial. Lancet Oncol 2011; 12: 575-582.

27. Tural D, Selcukbiricik F, Yildiz O, Elcin O, Erdamar S, Guney S, Demireli F, Buyukunal E, Serdengecti S. Preoperative versus postoperative chemoradiotherapy in stage T3, N0 rectal cancer. Int J Clin Oncol 2014; 19: 889-896.

28. Bosset JF, Calais G, Mineur L, Maingon P, StojanovicRundic S, Bensadoun RJ, Bardet E, Beny A, Ollier JC, Bolla M, Marchal D, Van Laethem JL, Klein V, et al. Fluorouracil-based adjuvant chemotherapy after preoperative chemoradiotherapy in rectal cancer: long-term results of the EORTC 22921 randomised study. Lancet Oncol 2014; 15: 184-190.
29. Wo JY, Mamon HJ, Ryan DP, Hong TS. T3N0 rectal cancer: radiation for all? Semin Radiat Oncol 2011; 21: 212-219.

30. Prolongation of the disease-free interval in surgically treated rectal carcinoma. Gastrointestinal Tumor Study Group. N Engl J Med 1985; 312: 1465-1472.

31. Fisher B, Wolmark N, Rockette H, Redmond C, Deutsch M, Wickerham DL, Fisher ER, Caplan R, Jones J, Lerner $\mathrm{H}$, et al. Postoperative adjuvant chemotherapy or radiation therapy for rectal cancer: results from NSABP protocol R-01. J Natl Cancer Inst 1988; 80: 21-29.

32. Gunderson LL, Callister M, Marschke R, Young-Fadok T, Heppell J, Efron J. Stratification of rectal cancer stage for selection of postoperative chemoradiotherapy: current status. Gastrointest Cancer Res 2008; 2: 25-33.

33. Nagtegaal ID, Tot T, Jayne DG, McShane P, Nihlberg A, Marshall HC, Pahlman L, Brown JM, Guillou PJ, Quirke P. Lymph nodes, tumor deposits, and TNM: are we getting better? J Clin Oncol 2011; 29: 2487-2492. 